\title{
Precise Knowledge on Presbyopic Contact Lenses
}

\section{Partha Haradhan Chowdhury ${ }^{1 *}$ and Brinda Haren Shah ${ }^{2}$}

1M. Optom, Department of Optometry, Shree Satchandi Jankalyan Samiti Netra

Prasikshan Sansthan, Pauri, Affiliated to Uttarakhand State Medical Faculty, Dehradun, India

Short Communication

Volume 3 Special Issue 1

Received Date: June 28, 2018

Published Date: July 20, 2018

2 M. Optom, Department of Optometry, Gujarat University, Gujarat, India

*Corresponding author: Partha Haradhan Chowdhury, M. Optom, Department of Optometry, Shree Satchandi Jankalyan Samiti Netra Prasikshan Sansthan, Pauri, Affiliated to Uttarakhand State Medical Faculty, Dehradun, India, Email: optometrypublish@gmail.com

\section{Abstract}

This study reveals about types of presbyopic contact lenses as well as its advantages and disadvantages.

Keywords: Presbyopic; Monovision; Contact Lenses

Before describing about presbyopic / bifocal contact lenses, it should be focused on its advantages and disadvantages [1-3].

\section{Advantages}

a. Patient will be free from spectacles.

b. Specatacle weight will be a considerable factor.

c. Cosmetically, contact lenses are better than spectacles.

\section{Disadvantages}
a. Reduced Stereo Acuity
b. Reduced Contrast Sensitivity
c. Fluctuation of vision
d. Changes in Binocular Balance

\section{Types of Presbyopic Contact Lenses}
A. Simultaneous vision Bifocals
B. Alternate vision Bifocals
C. Monovision
D. Modified Monovision
E. Enhanced Monovision
F. Diffractive Bifocals

\section{Simulataneous Vision Bifocals}

The meaning of Simulateous vision is, light will enter in each eye and origination of light will be from the distance and near object. In this case, when patient is asked to look for distant object, at that time, near object will be blurred and when asked to look for near object, distance object will be blurred.

It consists of 3 designs:

- Concentric design

- Aspheric design

- Diffractive design

Routinely, to improve visual functions, Hybrid design is considerable. Main disadvantage of this design is Reduced vision Quality

\section{Alternate Vision Bifocals}

Here, by changing the contact lenses, patient is able to see distant and near object. When patient is trying to see at near object, lower part of the contact lenses will be placed in front of the pupil.

\section{Monovision}

Here, one eye is used for distance purpose and another eye is used for near purpose. 


\section{Open Access Journal of Ophthalmology}

\section{Modified Monovision}

Here, bifocals are placed in both the eyes. Bifocal of one eye is used for distance purpose and bifocal of another eye is used for near purpose.

\section{Enhanced Monovision}

Here, single vision lens is placed in one eye and in another eye, bifocal contact lens is placed.

\section{Diffractive Bifocals}

Here, Huygen's principle (Light Wave Theory) is applied. Diffractive contact lenses come under Simultaneous Vision. This design consists of several zones. Zones are progressively increased. Here, distance and near power is placed at the optic zone but distant and near power is not placed on the pupillary zone.

\section{References}

1. Iacle Module $8,1^{\text {st }}(\mathrm{Edn})$.

2. Edward SB, Weissman BA (2005) Clinical Contact Lens Practice.

3. Mark J Mannis, Karla Zadnik, Cleusa Coral-Ghanem, Newton Kara-José (2006) Contact Lenses in Ophthalmic Practice. 